\title{
The boundary element method and path-independent integrals in sensitivity analysis of structures with stress concentration
}

\author{
T. Burczyński ${ }^{1,2}$ and M. Habarta ${ }^{1}$ \\ ${ }^{1}$ Department for Strength of Materials and Computational Mechanics, Silesian \\ University of Technology, Poland \\ ${ }^{2}$ Institute of Computer Modelling, Cracow University of Technology, Poland \\ e-mail: burczyns@polsl.gliwice.pl,mhabarta@rmt4.kmt.polsl.gliwice.pl
}

\begin{abstract}
This paper presents the implementation of the boundary element method to shape sensitivity analysis of elastic structures with stress concentrators. An elastic body which contains a number of voids (internal boundaries), playing the role of stress concentrators, is considered. We are interested in calculating the first order sensitivity of shape-dependent functionals with respect to the shape variation of the body domain. This task is accomplished using the adjoint variable method. As it has been shown by Dems and Mróz [10], for a basic transformation (i.e. a translation, a rotation or a scale change) of the body, the sensitivity of the considered functional takes the form of a path-independent integral (PII) whose integrand depends on the primary and adjoint state fields, along an arbitrary path (curve in $2 \mathrm{D}$, surface in $3 \mathrm{D}$ ), enclosing the transformed stress concentrator (void). It is very important for numerical computations, because we can compute this integral along the path placed far from the stress concentrator to eliminate the negative influence of stress concentrations on the accuracy of calculations. The boundary element method (BEM) is used to solve both primary and adjoint problems. Some important cases of adjoint problems related to functionals are analyzed in the paper. A thorough numerical verification of the proposed method is performed in this work. The presented method of sensitivity analysis is utilised in gradient-based optimization and identification problems. Numerical examples of optimization and identification are shown in this paper.
\end{abstract}

\section{Introduction}

The phenomenon of stress concentrations causes serious problems for engineers designing mechanical structures. As widely known, the reason of this phenomenon is usually the shape of the considered structure - stress concentrations occur in the vicinity of notches, voids, cracks, material discontinuities. In numerical analysis of elastic bodies, stress concentrations are often responsible for the drastic increase of numerical errors. It may be also observed in the case of sensitivity analysis, where not only stress or displacement gradients, but also stress derivatives or second order of displacement derivatives are computed. This paper presents a new method of shape sensitivity analysis which overcomes difficulties arisen from stress concentrations. The method is based on the adjoint variable approach and uses the boundary element method (BEM) for solving both primary and adjoint problems. To decrease numerical error resulting from stress concentrations the idea of path independent integrals is employed.

The analytical formulation of this concept was elaborated by Dems and Mróz in [10] who discussed a new class of conservation rules which constituted an extension of the 
class considered by Rice [11] and Bui [3]. The idea of coupling path-independent integrals and the boundary element method to shape sensitivity analysis and identification associated with singular and quasi-singular boundary variations was proposed by Burczyński [12] and next extended to crack problems by Burczy ński and Polch [2, 1] (see also [8]) in deterministic cases and by Burczyński $[5,4]$ for stochastic problems. Now, we develop this idea for shape sensitivity analysis of voids using boundary element procedures.

\section{Invariant integrals in shape sensitivity analysis}

Let $\Omega \subset \mathbb{R}^{d},(d=2$ or 3$)$ be a domain occupied by an elastic body with a boundary $\Gamma$. The body is assumed to be loaded only by boundary tractions $\boldsymbol{p}^{0}$ on the part $\Gamma_{p}$ of its boundary (no body forces occur). As usually, boundary displacements $\boldsymbol{u}^{0}$ are prescribed on the rest of the boundary $-\Gamma_{u}$.

We are interested in calculating the first order sensitivity of a shape-dependent functional of the general form

$$
J(\Omega)=\int_{\Omega} \Psi(\boldsymbol{\sigma}, \boldsymbol{u}) d \Omega+\int_{\Gamma} h(\boldsymbol{p}, \boldsymbol{u}) d \Gamma
$$

with respect to the shape variation of the domain $\Omega$, where integrands $\Psi$ and $h$ are arbitrary differentiable functions of their arguments.

To accomplish this task we use the adjoint variable method. In this method we introduce a new boundary problem - the so called adjoint problem - determined over the same domain $\Omega$ with the same material constants, but with different boundary and loading conditions :

$$
\boldsymbol{p}^{a}=\frac{\partial h}{\partial \boldsymbol{u}} \quad \text { on } \Gamma_{p}, \quad \boldsymbol{u}^{a}=-\frac{\partial h}{\partial \boldsymbol{p}} \quad \text { na } \Gamma_{u}
$$

and

$$
\boldsymbol{f}^{a}=\frac{\partial \Psi}{\partial \boldsymbol{u}}, \quad \varepsilon^{i a}=\frac{\partial \Psi}{\partial \boldsymbol{\sigma}} \quad \text { inside } \Omega
$$

If the functional $J$ contains the domain integral of the stress-dependent function, the adjoint system is loaded with initial strains.

The shape variation of the domain $\Omega$ is introduced in the form of a transformation $\varphi: \Omega \mapsto \mathbb{R}^{d}$

$$
\boldsymbol{x}^{*}=\varphi(\boldsymbol{x})=\boldsymbol{x}+f(\boldsymbol{x})
$$

where $\boldsymbol{x}^{*}$ denotes the transformed position of a material point $\boldsymbol{x} \in \Omega$, and $f-$ an infinitesimally small (in the sense of $C^{1}(\Omega)$ norm) transformation. Additionally, variations of boundary conditions must be determined in a way dependent on the shape transformation $f$, i.e. $\delta \boldsymbol{p}^{0}$ must be prescribed on $\Gamma_{p}$ and $\delta \boldsymbol{u}^{0}$ must be prescribed on $\Gamma_{u}$. 
Under above assumptions, a variation of the functional $\mathbf{J}$ defined by (1) can be expressed as

$$
\begin{aligned}
\delta J & =\int_{\Gamma}\left(\Psi \delta_{k j}+\sigma_{i j}^{r} u_{i, k}-u_{i, l}^{a} \sigma_{i l} \delta_{k j}+u_{i, k}^{a} \sigma_{i j}\right) n_{j} f_{k} d \Gamma \\
& +\int_{\Gamma}\left[h\left(\delta_{k l}-n_{k} n_{l}\right)-u_{i}^{a} \sigma_{i j} n_{j} n_{l} n_{k}+u_{i}^{a} \sigma_{i j} n_{j} \delta_{k l}\right] f_{k, l} d \Gamma \\
& +\int_{\Gamma_{u}}\left(\frac{\partial h}{\partial u_{i}}-\sigma_{i j}^{r} n_{j}\right) \delta u_{i} d \Gamma_{u}+\int_{\Gamma_{p}}\left(\frac{\partial h}{\partial p_{i}}+u_{i}^{a}\right) \delta p_{i} d \Gamma_{p}
\end{aligned}
$$

where $\sigma^{r}=C\left(\varepsilon^{a}-\varepsilon^{i a}\right)$. This formula contains boundary integrals with integrands containing displacement derivatives. It may result in significant growth of numerical errors, when integration is performed in the vicinity of stress concentrators. Dems and Mróz have shown in [10] that under some additional assumptions on functions $\Psi$ and $h$, equation (5) can be expressed (for a special set of shape transformations) in a form of a path independent integral, which allows for integrating far from the stress concentrations and consequently, decrease the numerical error.

\subsection{Particular cases of shape transformation}

Consider three cases of the general transformation given by (4): the translation, the rotation and the scale change.

Translation (T) The translation (either finite or infinitesimal) can be determined by a vector $\boldsymbol{a}$ as

$$
\boldsymbol{x}^{*}=T_{\boldsymbol{a}} \boldsymbol{x}=\boldsymbol{x}+\boldsymbol{a}
$$

Rotation (R) In this case we must distinguish between the finite and the infinitesimal rotation. Both can be parameterized by a vector $\boldsymbol{\omega}$ in $3 \mathrm{D}$. An infinitesimal rotation is given by

$$
\boldsymbol{x}^{*}=R_{\boldsymbol{\omega}} \boldsymbol{x}=\boldsymbol{x}+\boldsymbol{\omega} \times \boldsymbol{x}
$$

In the plane case, by putting $\boldsymbol{\omega}=(0,0, \alpha)$, this formula takes a simpler form

$$
x_{1}^{*}=x_{1}-\alpha x_{2}, x_{2}^{*}=x_{2}+\alpha x_{1}
$$

Expansion or scale change (E) An infinitesimal expansion is determined (for both spatial and plane cases) by a single number $r$ as

$$
\boldsymbol{x}^{*}=E_{r} \boldsymbol{x}=\boldsymbol{x}+r \boldsymbol{x}=\boldsymbol{x}(1+r)
$$

Assume, that the body under consideration occupies a multiple connected domain $\Omega$ with a boundary $\partial \Omega=\Gamma=\bigcup_{i=0}^{n} \Gamma_{i}$, where $\Gamma_{0}$ is the external boundary and $\Gamma_{i}$ for $i>0$ are internal boundaries. Let the external boundary undergoes the transformation given by (4), where $\varphi$ is one of the three kinds of transformation: the translation (T), the 
rotation $(\mathrm{R})$ or the scale change (expansion E). The boundary conditions prescribed on the $\Gamma_{0}$ are also transformed correspondingly (in the way described in [10]). From now on we make the following

Assumption 1. In definition (1) $\Psi$ and $h$ are isotropic and homogeneous functions of their arguments ${ }^{1}$.

According to [10], the variation of the functional given by (1) can be expressed in this case as a particular form of (5), namely

$$
\delta J=b_{k}\left(\mathcal{Z}_{k}^{\varphi}\right)_{\Gamma_{0}}=b_{k} \int_{\Gamma_{0}} Z_{k}^{\varphi}\left(\boldsymbol{u}, \boldsymbol{\sigma}, D \boldsymbol{u}, \boldsymbol{u}^{a}, \boldsymbol{\sigma}^{a}, D \boldsymbol{u}^{a}\right) d \Gamma_{0}
$$

where $b_{k}$ are parameters related to specific transformations $\varphi=T, R, E$. Integrands $Z_{k}^{\varphi}$ depend on the state fields of primary and adjoint solutions and take form:

-for the translation:

$$
Z_{k}^{T}=\left[\Psi \delta_{k j}+\sigma_{i j}^{r} u_{i, k}+\sigma_{i j} u_{i, k}^{a}-\sigma_{p q} \varepsilon_{p q}^{a} \delta_{k j}\right] n_{j}
$$

-for the rotation

$$
\left.Z_{p}^{R}=e_{k p l}\left(\Psi \delta_{k j}+\sigma_{i j}^{r} u_{i, k}-u_{i, l}^{a} \sigma_{i l} \delta_{k j}+u_{i, k}^{a} \sigma_{i j}\right) x_{l}+\sigma_{l j} u_{k}^{a}+\sigma_{l j}^{r} u_{k}\right) n_{j}
$$

-for the expansion

$$
Z^{E}=\left[\left(\Psi \delta_{j l}+\sigma_{i j}^{r} u_{i, l}-u_{i, p}^{a} \sigma_{i p} \delta_{l j}+u_{i, k}^{a} \sigma_{i j}\right) x_{l}-\xi \sigma_{i j}^{r} u_{i}+(\xi-2+d) u_{i}^{a} \sigma_{i j}\right] n_{j}
$$

where $d$ denotes the dimensionality of the problem and $\xi$ is the number depending on the form of the functional (see [10]). It has been proved in [10] that under the assumption 1

$$
\left(\mathcal{Z}_{k}^{\varphi}\right)_{S}=\int_{S} Z_{k}^{\varphi}\left(\boldsymbol{u}, \boldsymbol{\sigma}, D \boldsymbol{u}, \boldsymbol{u}^{a}, \boldsymbol{\sigma}^{a}, D \boldsymbol{u}^{a}\right) d S=0
$$

for any surface (curve) $S$ within the body that encloses a homogeneous domain. It means, that integrals $(\mathcal{Z})_{S}$ are path-independent.

\subsection{Application of $(\mathcal{Z})_{S}$ integrals to sensitivity analysis of stress con- centrators}

In the above considerations it was assumed, that the external boundary was transformed. However, it is equivalent to the transformation $\varphi^{-1}$ of an internal boundary (void) with the external boundary being unchange (Fig. 1). Suppose, that we want to compute the variation of $J$ with respect to the transformation $\psi$ of only one part of the internal boundary, say $\Gamma_{1}$ (Fig. 1). Enclose $\Gamma_{1}$ in a surface $S_{0}$, so that other voids are left on its other side (Fig. 1). Introduce $\Gamma_{0}^{\prime}=\Gamma \backslash \Gamma_{1}$ as the external boundary undergoing the equivalent transformation $\varphi=\psi^{-1}$. Then, sensitivity of $J$ with respect to the transformation

\footnotetext{
${ }^{1}$ In fact, even more specific form of $J$ is required when sensitivity with respect to the expansion is considered, see [10] for details.
} 


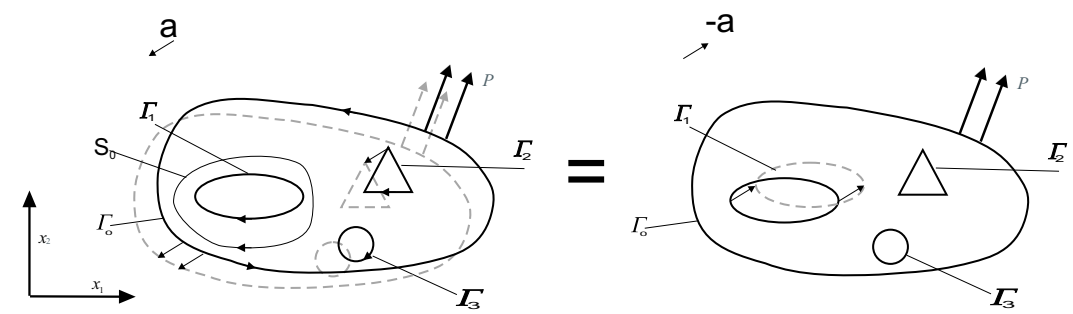

Figure 1: Boundary transformation

of the void $\Gamma_{1}$ equals $\frac{D J}{D \varphi}=(\mathcal{Z})_{\Gamma_{0}^{\prime}}$. On the other hand $S=S_{0} \cup \Gamma_{0}^{\prime}$ is the boundary of a homogeneous (without voids) and isotropic domain included in $\Omega$, thus, taking into consideration (14) we have $0=(\mathcal{Z})_{S}=(\mathcal{Z})_{S_{0} \cup \Gamma_{0}^{\prime}}=(\mathcal{Z})_{S_{0}}+(\mathcal{Z})_{\Gamma_{0}^{\prime}}$ and consequently, $(\mathcal{Z})_{\Gamma_{0}^{\prime}}=-(\mathcal{Z})_{S_{0}}$. Since $\varphi=\psi^{-1}$, we have $\frac{D J}{D \varphi}=-\frac{D J}{D \psi}$ and finally

$$
\frac{D J}{D \psi}=(\mathcal{Z})_{S_{0}}
$$

It means, that the sensitivity of the functional $J$ is expressed by the path-independent integral along any arbitrary surface (or contour for 2D problems) $S_{0}$ enclosing the stress concentrators.

\section{Boundary element method in shape sensitivity analysis}

In order to perform sensitivity analysis one should solve two direct problems: primary and adjoint problems. For solving both primary and adjoint problems the boundary element method (BEM) is used. In this method we discretise and solve a boundary integral equation of the form

$$
c_{l k} u_{k}^{w}+\int_{\Gamma} P_{l k}^{*} u_{k}^{w} d \Gamma=\int_{\Gamma} U_{l k}^{*} p_{k}^{w} d \Gamma+\int_{\Omega} U_{l k}^{*} b_{k}^{w} d \Omega
$$

where $w=p$ for the primary problem, $w=a$ for the adjoint problem and $U_{l k}^{*}, P_{l k}^{*}$ are the fundamental Kelvin solutions. According to this notation $b_{k}^{p}=0$ and $b_{k}^{a}$ depends on the initial strain field associated with the integrand $\Psi$.

\subsection{Problems with initial strains}

To solve the adjoint problem given, by (2) and (3), one should introduce initial strains into the domain. This can be cumbersome and may diminish some advantages of boundary element method.

In problems with initial strains $\varepsilon^{i}$ the field of total strains $-\varepsilon$, which is compatible with the displacement field by $\varepsilon_{k l}=\frac{1}{2}\left(u_{k, l}+u_{l, k}\right)$, satisfies equation

$$
\varepsilon=\varepsilon^{i}+\varepsilon^{r}
$$


where $\varepsilon^{r}$ denotes the field of elastic strains related to the stress tensor $\sigma^{r}$ by the Hooke's law $\boldsymbol{\sigma}^{r}=C \cdot \varepsilon^{r}$ and thus indirectly connected with the body forces by the equilibrium condition $\operatorname{div} \boldsymbol{\sigma}^{r}+\boldsymbol{b}=0$

One of the most widely used method of solving such problems is the fictitious force method, which has been used in the present work. In this method, we transform problem with initial strains into the problem with body forces. Defining modified force field $\boldsymbol{b}^{*}=$ $-\operatorname{div} \boldsymbol{\sigma}^{i}+\boldsymbol{b}$, where $\boldsymbol{\sigma}^{i}=C \boldsymbol{\varepsilon}^{i}$ is the initial stress, the Navier's equation is obtained

$$
L(\boldsymbol{u})+\boldsymbol{b}^{*}=0
$$

where $L(u)$ is the Lame operator. Additionally we must modify the boundary conditions stating $\boldsymbol{p}^{*}=\boldsymbol{p}+\boldsymbol{\sigma}^{i} \cdot \boldsymbol{n}$ as the traction field.

\subsection{Particular cases of adjoint problems}

Here we present some cases of adjoint problems generated by particular forms of the functional $J$.

\subsubsection{Functionals depending on boundary displacements}

One of the simplest of them is the functional given by

$$
J=\int_{\Gamma} h(\boldsymbol{u}) d \Gamma=\int_{\Gamma}[\boldsymbol{p}(x) \cdot \boldsymbol{u}(x)] \delta\left(x-x_{0}\right) d \Gamma(x)=\boldsymbol{p}\left(x_{0}\right) \cdot \boldsymbol{u}\left(x_{0}\right)
$$

which, assuming that $\left|\boldsymbol{p}\left(x_{0}\right)\right|=1$, expresses the projection of the displacement at given boundary point $\boldsymbol{x}_{0}$ on the direction determined by vector $\boldsymbol{p}$. The adjoint system for such a functional is loaded only with a concentrated force $\boldsymbol{p}$ applied at point $\boldsymbol{x}_{0}$.

\subsubsection{Functionals depending on internal stresses}

These functionals are defined as $J=\int_{\Omega} \Psi(\sigma) d \Omega$. As we noticed in the previous section, the invariance of integrals (11)-(13) is guaranteed under the assumption that $\Psi$ and $h$ are isotropic and homogeneous functions of their arguments.

This leads to the general form of $\Psi$ as

$$
\Psi(\boldsymbol{\sigma})=g\left(\frac{1}{2} \cdot \boldsymbol{\sigma} D \boldsymbol{\sigma}\right)
$$

where $g$ is a homogeneous of order $p, C^{1}$ function and $D$ is an isotropic tensor. Due to the homogenity condition we have $g(t)=g(1) t^{p}$. On the other hand, isotropy of $D$ yields

$$
D \boldsymbol{\sigma}=a \boldsymbol{\sigma}+b \operatorname{Tr}(\boldsymbol{\sigma}) I
$$

Now we are going to concentrate on three general cases of stress functionals. 
Complementary energy. Under the condition, that only the surface tractions expend the work on the body, the functional $J$ may represent the complementary energy. This is the case, where $g(t)=t$ and tensor $D$ is the one from the inverse Hooke's law : $D \sigma=\varepsilon$. This functional can be expressed as a boundary integral due to $\int_{\Omega} \boldsymbol{\sigma} \varepsilon d \Omega=\int_{\Gamma} \boldsymbol{u p} d \Gamma$, which is a very convenient property. In the adjoint problem we have $\varepsilon^{a i}=\varepsilon$ and thus $\boldsymbol{\sigma}^{i a}=C \boldsymbol{\varepsilon}=\boldsymbol{\sigma}$. Moreover, it is easy to check that $\boldsymbol{u}^{a}=\boldsymbol{u}$. Thus, having solved the primary system we have already known all the fields of the adjoint system.

Integral of the square of equivalent stress In this case $\Psi(\sigma)=\frac{1}{2} \cdot \sigma D \sigma$. Initial strains in the adjoint system are given as $\varepsilon^{i a}=D \sigma$. For particular values of $a, b$ in (21) we can get $\Psi(\boldsymbol{\sigma})=\boldsymbol{\sigma}_{e q}^{2}$ where $\boldsymbol{\sigma}_{e q}$ is the Huber - von Mises equivalent stress. Let us prove:

Theorem 1. The field of the fictitious body forces related to the initial stress in the adjoint system of the functional $J=\int_{\Omega} \frac{1}{2} \boldsymbol{\sigma} \cdot D \boldsymbol{\sigma} d \Omega$ where $D$ is given by (21) is the gradient of a harmonic potential.

Proof. Substituting Hooke's law $\sigma^{i}=2 \mu \varepsilon^{i}+\lambda \varepsilon^{i}$ into (21) we obtain $\sigma^{i}=2 \mu \boldsymbol{\sigma}+$ $(2 \mu b+\lambda a+\lambda b \operatorname{Tr}(I)) \operatorname{Tr}(\boldsymbol{\sigma}) I$ which may be rewritten as

$$
\boldsymbol{\sigma}^{i}=c \boldsymbol{\sigma}+d \operatorname{Tr}(\boldsymbol{\sigma}) I
$$

where $c=2 \mu, d=2 \mu b+\lambda a+2 \lambda b$. Calculating divergence of both sides of (22) we get

$$
\operatorname{div} \boldsymbol{\sigma}^{i}=c \operatorname{div} \boldsymbol{\sigma}+d \operatorname{div}[\operatorname{Tr}(\boldsymbol{\sigma}) I]
$$

But $\sigma$ is the stress field in the primary system, where body forces do not exist and thus $\operatorname{div} \boldsymbol{\sigma}=0$. On the other hand, it is well known, that in such a case $\Delta \operatorname{Tr}(\boldsymbol{\sigma})=0$ i.e. $\operatorname{Tr}(\boldsymbol{\sigma})$ is a harmonic field. Thus, denoting by $\boldsymbol{b}^{i}$ the fictitious force field in the adjoint system we can state

$$
\boldsymbol{b}^{i}=-\operatorname{div} \boldsymbol{\sigma}^{i}=-d \operatorname{grad} \operatorname{Tr}(\boldsymbol{\sigma})
$$

which completes the proof.

This theorem is very important from the numerical point of view as it allows to transform the volume integral $\int_{\Omega} U_{l k} b_{k} d \Omega$ into the boundary integral using the Galerkin vector $G_{k l}=\frac{1}{8 \pi \mu} r \delta_{k l}$ for 3D problems and $G_{k l}=-\frac{1}{8 \pi \mu} r^{2} \ln (r) \delta_{k l}$ for 2D case (see [7] for details).

In the case of the complementary energy we were able not only to simplify the solution of the adjoint problem, but also the value of the functional could be itself expressed as the boundary integral. Following theorem states, that, it is also possible in this case.

Theorem 2. If tensor $D$ is of the form (20), then

$$
\int_{\Omega} \boldsymbol{\sigma} D \boldsymbol{\sigma} d \Omega=\int_{\Gamma} \boldsymbol{p} \boldsymbol{u}^{a} d \Gamma
$$


Proof. We have $2 J=\int_{\Omega} \boldsymbol{\sigma} D \boldsymbol{\sigma} d \Omega=\int_{\Omega} \boldsymbol{\sigma} \varepsilon^{i} d \Omega=\int_{\Omega} \boldsymbol{\sigma}\left(\varepsilon^{i}-\varepsilon^{r}\right) d \Omega=\int_{\Omega} \sigma_{i j} u_{i, j}^{a} d \Omega-$ $\int_{\Omega} \sigma_{i j}^{r} u_{i, j} d \Omega$. The last integrals can be rewritten using the Gauss theorem as

$\int_{\Omega} \sigma_{i j} u_{i, j}^{a} d \Omega-\int_{\Omega} \sigma_{i j}^{r} u_{i, j} d \Omega=-\int_{\Omega} \sigma_{i j, j} u_{i}^{a} d \Omega+\int_{\Gamma} p_{i} u_{i}^{a} d \Gamma+\int_{\Omega} \sigma_{i j, j}^{r} u_{i} d \Omega-\int_{\Gamma} p_{i}^{r} u_{i} d \Gamma$

Since $\sigma_{i j, j}^{r}=0$ and $\frac{\partial h}{\partial u}=p_{i}^{r}=0$ the last equality proves (25).

\subsubsection{Integrals of higher powers of equivalent stresses}

Setting $g(t)=t^{p}$ in (20) we obtain

$$
J_{p}=\int_{\Omega}\left(\frac{1}{2} \cdot \boldsymbol{\sigma} D \boldsymbol{\sigma}\right)^{p} d \Omega=\int_{\Omega} \sigma_{e q}^{2 p} d \Omega
$$

This functional is connected with well known in functional analysis the $L_{p}$ norm of a function defined as $\|f\|_{p}=\left(\int_{\Omega}|f|^{p} d \Omega\right)^{1 / p}$, for $p \geq 1$. This norm has a very convenient property: if $|\Omega|<\infty$ and $f$ is bounded, then $\lim _{p \rightarrow \infty}|| f \|_{p}=\max |f(x)|$

This allows for approximation of nondifferentiable functional $\max \boldsymbol{\sigma}_{e q}$ by differentiable $J_{p}$. However, there are some limitations for the maximum exponent $p$. The problem is, that for cases where the boundary $\Gamma$ contains notches, the field $\boldsymbol{\sigma}_{e q}^{2}$ is not bounded. It follows from the Williams expansion (see $[15,14]$ ), that in such cases $\sigma_{i j}$ is singular of the type $O\left(r^{\lambda}\right)$, and $-1 / 2 \leq \lambda<0$. Then, the order of singularity of the field $\sigma_{e q}^{2 p}$ is $2 \lambda p$, thus the condition of its integrability yields $2 \lambda p>-2$, which gives

$$
p<-1 / \lambda
$$

Consider now the adjoint system generated by the functional (26). Initial strains are given by

$$
\varepsilon^{i}=\partial_{\boldsymbol{\sigma}} \Psi=p\left(\frac{1}{2} \boldsymbol{\sigma} D \boldsymbol{\sigma}\right)^{p-1} D \boldsymbol{\sigma}
$$

This implicates that the field of initial strain and stress has the singularity $O\left(r^{2 p \lambda-\lambda}\right)$. Hence, the field of fictitious forces $b_{j}^{*}=\sigma_{k j, k}^{i}$ is singular of type $O\left(r^{2 p \lambda-\lambda-1}\right)$. As the expression $U_{l k}^{*}(x, y) b_{k}(y)$ must be integrable, the order of singularity of $\boldsymbol{b}$ must be smaller than $O\left(r^{-2}\right)$ (in 2D) which yields $2 p \lambda-\lambda-1>-2$ and finally

$$
p<\frac{1}{2}\left(1-\frac{1}{\lambda}\right)
$$

Note, that inequality (29) is more restrictive than (27) if $\lambda \in\left[-\frac{1}{2}, 0\right)$. If only the former of them is fulfilled, then the functional is properly defined but the related adjoint system cannot be solved using BEM and fictitious forces method. In the worst case of stress concentration - the crack, $\lambda=-\frac{1}{2}$ and (29) yields that $p<\frac{3}{2}$ 


\subsection{Numerical implementation}

Practical calculating of $\left(\mathcal{Z}^{\varphi}\right)_{S}$ integrals is straightforward. First, we solve the primary problem and using obtained results generate data for the adjoint system. After having solved the latter, we create a contour $S$ enclosing the analysed void (internal boundary). This contour is divided into a finite number of curvilinear segments $S=\bigcup_{i=1}^{n_{s}} S_{i}$, each of them parameterized by $s_{i}: I \mapsto S_{i}$, where $I=[-1,1]$. Then we integrate the functions (11)-(13) along segments $S_{i}$ in the standard way: $\int_{S_{i}} \boldsymbol{z}(x) d S_{i}(x)=$ $\int_{I} \boldsymbol{z}\left(s_{i}(\xi)\right)\left|s_{i}^{\prime}(\xi)\right| d I(\xi)$, where the last integral is computed numerically using 10-point Gauss quadrature.

\section{Numerical tests in sensitivity analysis}

The aim of this section is to compare sensitivities computed using the path-independent integral method (denoted as PII) to these obtained by other numerical method of sensitivity analysis. One of these methods (the finite difference method denoted as FD) gives approximation of the sensitivity of the functional $J(x)$ (depending on one argument) $x$ as $\frac{\mathrm{d} J}{\mathrm{~d} x} \approx \frac{J(x+h)-J(x)}{h}$. This is simple but may be very sensitive to accuracy of computing $J(x)$. Hence we used more refined technique: instead of computing values of analysed functional at only two points, we construct the least-squares approximation of $J$ in the vicinity of $x$ - a polynomial of 2-nd degree is used as approximating function. If the functional is expressed in the form of a volume integral this method is referred to as API, in the case of a boundary integral - APB.

In all examples which will be presented, the material constants are set as $G=8$ and $\nu=0.2$. The program used to compute them accepted dimensionless ${ }^{2}$ data, so the unit choice was implicitly included in the preparing data and the result interpretation .

Example 1. A rectangular plate under concentrated force was considered (Fig.2(b)) with various shapes of internal void (see also [9],[13]). The functional being analysed is of

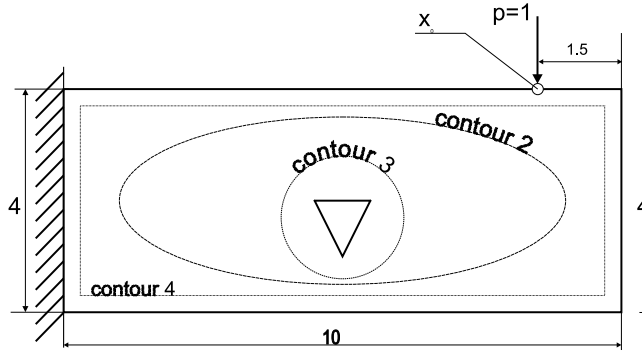

(a) for various integration paths

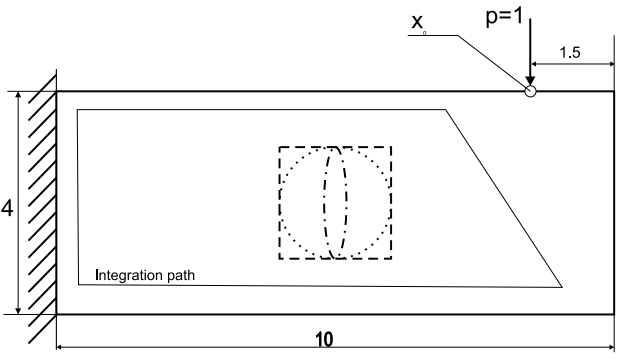

(b) for various voids

Figure 2: Tests for displacement functional (Example 1)

the form (19) and expresses a vertical displacement of the $x_{0}$. Results shown in table 1

\footnotetext{
${ }^{2}$ Note, that we consider linear problems.
} 


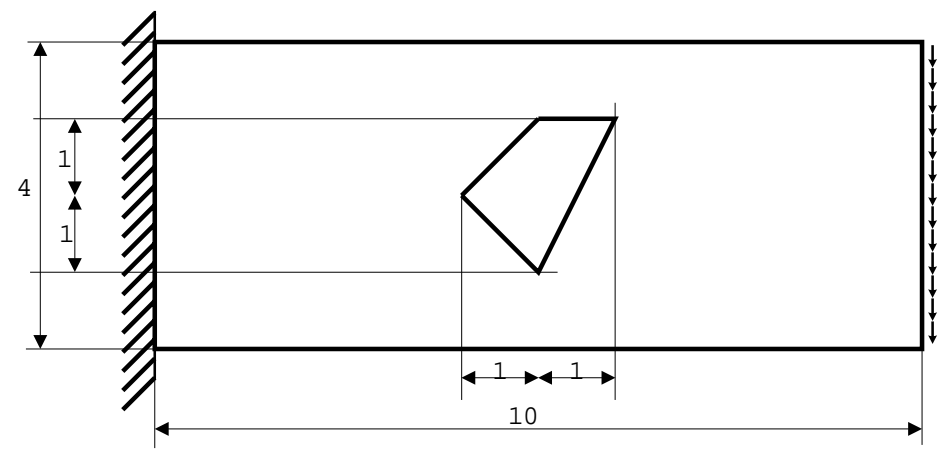

Figure 3: Rectangular plate with a tetragonal void (Example 2)

have a very good agreement. Then, for the triangular void (Fig. 2(a)) integrals PII

Table 1: Sensitivities of $J$ for various shapes of voids (ex. 1)

\begin{tabular}{||l||l|l|l|l|l||}
\hline \hline Void shape & Method & $\mathrm{Tx} \times 10^{2}$ & $\mathrm{Ty} \times 10^{2}$ & Rot $\times 10^{2}$ & Exp \\
\hline \hline \multirow{2}{*}{ circle $(\mathrm{r}=1, \mathrm{O}=(0,0))$} & PII & -0.1723 & -0.1613 & $-0.5 \mathrm{e}-5$ & 0.1040 \\
& FD & -0.1759 & -0.1583 & $0.3 \mathrm{e}-4$ & 0.1040 \\
\hline ellipse $($ width $\mathrm{x}=0.2$, & PII & -0.1025 & -0.0545 & 0.0152 & 0.0229 \\
width $\mathrm{y}=1 \mathrm{O}=(0,0))$ & FD & -0.1154 & -0.0529 & 0.0144 & 0.0237 \\
\hline triangle (vert.: $\quad(-0.5,0)$ & PII & -0.0572 & -0.3618 & 0.1374 & 0.0125 \\
$(0.5,0)(0,-1)$ & FD & -0.0615 & -0.3753 & 0.1362 & 0.0126 \\
\hline square $(\mathrm{O}=(0,0)$, vert.:( & PII & -0.2583 & -0.2889 & -0.1710 & 0.3050 \\
$1,1))$ & FD & -0.2725 & -0.2832 & -0.1679 & 0.3043 \\
\hline \hline
\end{tabular}

were computed along various paths. Results in Table 2 provide a confirmation of the correctness of the calculations - they should be equal - and they are approximately equal.

Table 2: Integrals PII for various integration contours (ex. 1)

\begin{tabular}{|c|c|c|c|c|}
\hline Contour & \multicolumn{1}{|c|}{$\mathrm{Tx} \times 10^{3}$} & $\mathrm{Ty} \times 10^{2}$ & Rot $\times 10^{2}$ & Exp $\times 10$ \\
\hline \hline 2 & -0.57195 & -0.361796 & 0.13744 & 0.124680 \\
\hline 3 & -0.57189 & -0.361800 & 0.13745 & 0.124681 \\
\hline 4 & -0.57192 & -0.361798 & 0.13744 & 0.124681 \\
\hline
\end{tabular}

Example 2. A rectangular plate shown in Fig. 3 with a tetragonal void is fixed on one side and loaded by a tangential distributed force of intensity 1 on the other side. First, consider the functional representing the complementary energy. This functional can be computed as a boundary integral $J_{\Gamma}=\frac{1}{2} \int_{\Gamma} \boldsymbol{p} \boldsymbol{u} d \Gamma$ as well as a volume integral $J_{\Omega}=\frac{1}{2} \int_{\Omega} \boldsymbol{\sigma} \varepsilon d \Omega$. From tests, which have been performed, it follows that computing the complementary energy as the volume integral is very time consuming compared to the former way. For example, when we divided the boundary into 64 elements the value of $J_{G}$ was computed 
in $35 \mathrm{~ms}$ and $J_{\Omega}$ - by dividing the interior of $\Omega$ into 480 triangles and using 7 point Gauss cubature - in $30 \mathrm{~s}^{3}$. It has been also noted, that calculation of $J_{\Gamma}$ is far more accurate that $J_{\Omega}$

Table 3 shows values of sensitivities of the complementary energy computed with the APB method for various discretizations of the boundary. The same calculations were

Table 3: Sensitivity of the complementary energy (computed as $\frac{1}{2} \int_{\Gamma} \boldsymbol{p} \boldsymbol{u} d \Gamma$ by the APB method Example 2)

\begin{tabular}{||c||r|r|r|r||}
\hline Num. of bound. elem. & translation $\mathrm{x}$ & translation $\mathrm{y}$ & rotation & expansion \\
\hline \hline 32 & -0.8618 & 0.4327 & 2.0118 & 7.1337 \\
\hline 64 & -0.2859 & 0.5705 & 2.4190 & 7.5603 \\
\hline 128 & -0.2699 & 0.5598 & 2.5306 & 7.6470 \\
\hline
\end{tabular}

performed using the volume integral as the value of this functional. Results are presented in Table 4. Then, PII method was used to obtain sensitivities. As the integration contour

Table 4: Sensitivity of the complementary energy (computed as $\frac{1}{2} \int_{\Omega} \sigma \varepsilon d \Omega$ by the API method Example 2)

\begin{tabular}{||c||r|r|r|r||}
\hline Number of boundary elements & translation x & translation y & rotation & expansion \\
\hline \hline 32 & -1.4859 & 0.2936 & 1.6148 & 6.8404 \\
\hline 64 & -0.2997 & 0.5950 & 2.2960 & 7.4657 \\
\hline 128 & -0.2690 & 0.5809 & 2.4793 & 7.6020 \\
\hline
\end{tabular}

we used the circle with the centre coinciding with te centre of the rectangle and the radius 1.7. Results are shown in Table 5

Table 5: Sensitivity of complementary energy obtained by PII method (Example 2 2).

\begin{tabular}{||c||r|r|r|r||}
\hline Num. of bound. elem. & translation $\mathrm{x}$ & translation $\mathrm{y}$ & \multicolumn{1}{c|}{ rotation } & expansion \\
\hline \hline 32 & -0.2728 & 0.6544 & 2.2076 & 7.2305 \\
\hline 64 & -0.2738 & 0.5936 & 2.4540 & 7.5409 \\
\hline 128 & -0.2708 & 0.5604 & 2.5417 & 7.6336 \\
\hline
\end{tabular}

From these comparison it follows that the least differences occur between results in Table 5 and 3. Integration over the interior of the domain (Table 4) gives also close results, but differences are greater, especially when the discretization is coarse. Nevertheless, we can state, that the API method is quite reliable in this case.

Integral of the square of equivalent stresses The value of this functional equals 1233.6. In Table 6 sensitivity coefficients obtained by the API method are shown for various boundary discretization. These results may be compared to the results obtained by the PII method, shown in table 7

\footnotetext{
${ }^{3}$ this does not include the time of solving boundary problem which was identical in both cases $-4.2 \mathrm{~s}$
} 
Table 6: Sensitivity of $\int_{\Omega} \sigma_{e q}^{2} d \Omega$ computed by the API method (Example 2)

\begin{tabular}{||c||r|r|r|r||}
\hline Num. of bound. elem. & translation $\mathrm{x}$ & translation $\mathrm{y}$ & rotation & expansion \\
\hline \hline 32 & -59.801 & 11.700 & 64.202 & 273.015 \\
\hline 64 & -12.051 & 23.740 & 91.923 & 298.777 \\
\hline 128 & -10.784 & 23.437 & 99.195 & 304.114 \\
\hline 210 & -10.736 & 22.321 & 101.758 & 306.198 \\
\hline
\end{tabular}

Table 7: Sensitivity of $\int_{\Omega} \sigma_{e q}^{2} d \Omega$ computed by the PII method (Example 2)

\begin{tabular}{||c||r|r|r|r||}
\hline Num. of bound. elem. & translation $\mathrm{x}$ & translation y & rotation & expansion \\
\hline \hline 32 & 1.56672 & 8.9914 & 144.398 & 309.841 \\
\hline 64 & -1.65994 & 13.5948 & 128.276 & 306.862 \\
\hline 128 & -3.63688 & 13.9315 & 125.658 & 310.968 \\
\hline 210 & -6.54046 & 15.8507 & 118.834 & 308.205 \\
\hline
\end{tabular}

In this case differences are considerably larger than for the complementary energy. It can be seen, that sensitivities obtained by both methods are getting closer as the boundary discretization is getting finer - supposedly, they converge to the common limit.

\section{Optimization and identification}

One of the most important application of sensitivity analysis is optimization and identification. Thus, we attempt to apply PII method to optimization problems. However, some additional difficulties should be overcome in this case.

First, we considered the single, infinitesimal translation, rotation or expansion. If we want to construct an optimization algorithm, we must determine the design variables and the way of calculation of the partial derivatives of the functional.

\subsection{Design variables}

Let us restrict ourselves to plane cases (for simplicity), but almost everything we will state can be easily generalized for $3 \mathrm{D}$ problems. As we have already mentioned, the translation depends on 2 variables, the rotation depends on 1, the expansion - on 1 variable. Note, that these three families of transformation generate the group ${ }^{4}$ of all affine transformations preserving angles ${ }^{5}$. It can be easily seen that each of such a transformation can be uniquely expressed as

$$
A_{\left(a_{1}, a_{2}, \alpha, r\right)}=T_{\left(a_{1}, a_{2}\right)} O_{\alpha} E_{r}
$$

i.e. as the superposition of the finite expansion $E_{r} \boldsymbol{x}=e^{r} \boldsymbol{x}$, the finite rotation $O_{\alpha}=$ $\left(\begin{array}{cc}1 & -\alpha \\ \alpha & 1\end{array}\right) \boldsymbol{x}$ and the translation $T_{\boldsymbol{a}} \boldsymbol{x}=\boldsymbol{a}+\boldsymbol{x}$ with the specified order. To prove, that

\footnotetext{
${ }^{4}$ Meant in the strict, algebraic sense.

${ }^{5}$ When identifying the plane with the field of complex numbers, they are conformal transformations $z \mapsto$ $a z+b$
} 
transformations of the form (30) constitutes a group, one can use the equality $A T_{\boldsymbol{a}}=$ $T_{A \boldsymbol{a}} A$ holding for any linear transformation $A$ and translation $T_{\boldsymbol{a}}$. Note that the expansion and the rotation used in (30) have the center in $\mathbf{0}$, but any finite rotation or expansion $\varphi$ with an arbitrary centre $\boldsymbol{x}_{0}$ can be expressed as a superposition of translation and the corresponding transformation $A$ with centre in the $\mathbf{0}$ according to $\varphi(x)=x_{0}+A(x-$ $\left.x_{0}\right)=x_{0}+A x_{0}+A x=T_{x_{0}+A x_{0}} A(x)$.

Now, we are at the position, to determine how the shape of the domain is connected with design variables $\left(a_{1}, a_{2}, \alpha, r\right)$. Let $\Gamma_{i}$ be an arbitrary internal boundary (void), this untransformed configuration will be treated as the reference configuration. The shape of the domain $\Omega$ determined by the vector $\left(a_{1}, a_{2}, \alpha, r\right)$ is the shape with $\Gamma_{i}$ transformed by $A_{\left(a_{1}, a_{2}, \alpha, r\right)}$ in (30) as $\Gamma_{i}^{\prime}=A_{\left(a_{1}, a_{2}, \alpha, r\right)} \Gamma_{i}$.

\subsection{Derivatives with respect to design variables}

Formulas (10)- (13) express sensitivities of the functional $J$ with respect to the single transformation. However, we need derivatives of this functional with respect to the variables $a_{1}, a_{2}, \alpha, r$. In order to obtain them, we must express the small increase of the parameter $v_{i}$ in the vector $\boldsymbol{v}=\left(a_{1}, a_{2}, \alpha, r\right)$ as the small transformation. Thus we have $T_{a} O_{\alpha+\delta \alpha} E_{r}=O_{\delta \alpha} T_{b} O_{\alpha} E_{r}$ where $b=O_{\delta \alpha}^{-1} a$ for the rotation parameter and $T_{a} O_{\alpha} E_{r+\delta r}=E_{\delta r} T_{-\delta r a} T_{a} O_{\alpha} E_{r}$ for the expansion parameter. This leads to expressions for partial derivatives of $J$ as

$$
\begin{aligned}
\frac{\partial J}{\partial a_{i}} & =\frac{D J}{D T_{a_{i}}}, \text { for } i=1,2 \\
\frac{\partial J}{\partial \alpha} & =\frac{D J}{D O_{\alpha}}+\frac{D J}{D T_{a_{1}}} a_{2}-\frac{D J}{D T_{a_{2}}} a_{1} \\
\frac{\partial J}{\partial r} & =\frac{D J}{D E_{r}}-\frac{D J}{D T_{a_{1}}} a_{1}-\frac{D J}{D T_{a_{2}}} a_{2}
\end{aligned}
$$

where $\frac{\partial J}{\partial a_{i}}, \frac{\partial J}{\partial \alpha}$ and $\frac{\partial J}{\partial r}$ denotes partial derivatives of $J$, whereas $\frac{D J}{D T_{a_{i}}}, \frac{D J}{D O_{\alpha}}$ and $\frac{D J}{D E_{r}}$ denotes sensitivities of the functional $J$ with respect to corresponding infinitesimal transformation. For more detailed derivation of (31)- (31) see [6].

\subsection{Choice of optimization algorithm}

PII method of sensitivity analysis may be applied in any optimization algorithm which uses the gradient of the optimized functional. In all examples presented in this paper, we used the conjugate gradient method. For constrained problems the penalty function method is applied.

\subsection{Examples of optimization}

Example 3. The plate shown on the Figure 4 has been optimized with respect to the following functionals

1. Complementary energy $J=\frac{1}{2} \int_{\Gamma} \boldsymbol{p u} d \Gamma$, 


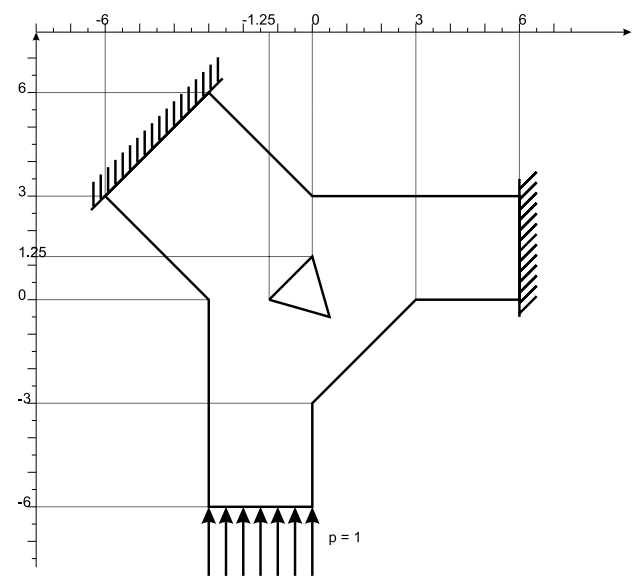

Figure 4: Structure from Example 3

2. Stress-dependent functional $J=\int_{\Omega} \boldsymbol{\sigma}_{e q}^{2} d \Omega$,

In all these cases there has been imposed the lower bound on the expansion parameter as $r \geq \ln 0.5$.

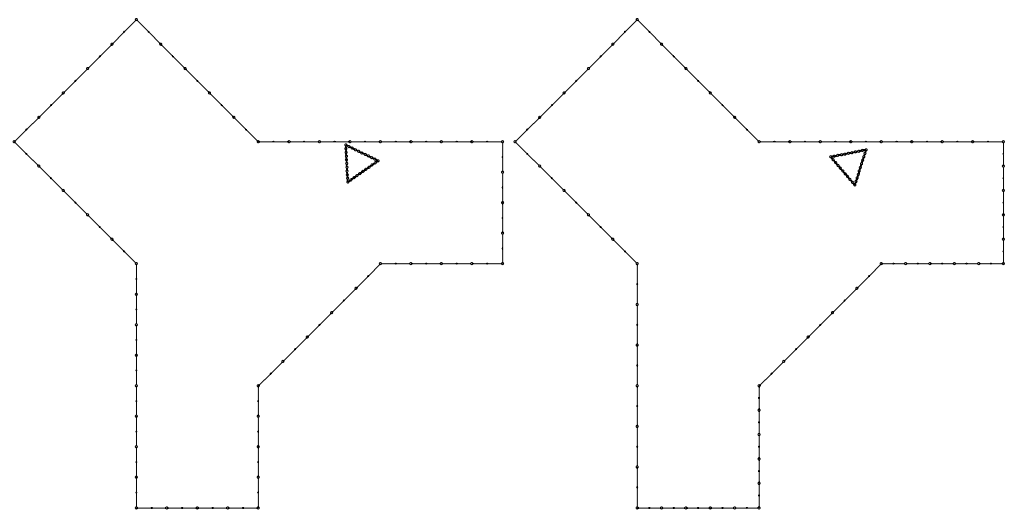

$\begin{array}{ll}\text { (a) After minimization of } \int_{\Omega} \sigma_{e q}^{2} d \Omega & \text { (b) After minimization of the complementary }\end{array}$ energy

Figure 5: Optimized structure from Example 3

\section{Minimization of the complementary energy}

Initial value :

Final value:

Calls of the objective function: 


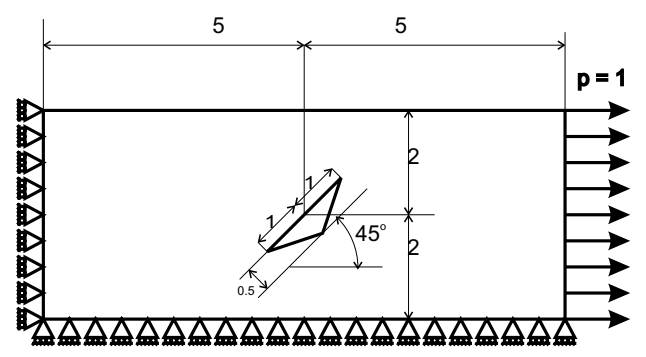

Figure 6: Structure from Example 4

Iterations of the main algorithm:

Computation time (Intel Celeron 433):

Final shape parameters:
10

$20 \mathrm{~min}$

$(2.281,2.280,-0.585,-0.69315(\ln 0.5)$

We can see that the lower bound for the expansion has been attained, which suggests that algorithm tended to make the void as small as possible to decrease the compliance of the structure.

Minimization of $\int_{\Omega} \sigma_{e q}^{2} d \Omega$

Initial value :

Final value:

Calls of the objective function:

154

Iterations of the main algorithm:

9

Computation time (Intel Celeron 433):

$5 \mathrm{~h}$

\section{Final shape parameters:}

$(2.353,2.326,-1.238,-0.69337(\ln 0.5)$

Example 4. A rectangular plate (Figure 6) with the triangular void is subjected to the tension load. The aim is to change the location of this void to minimize stresses in the plate. For that purpose minimization of the functional $J_{p}=\int_{\Omega} \sigma_{e q}^{3} d \Omega$ (thus $p=1.5$ ) is performed. Additionally, the size of the void cannot be decreased (i.e. $r>0$ ).

\section{Start:}

Initial value of $J_{p}$ :

Fianal value of $J_{p}$ :

Calls of the objective function :

Iterations of the main algorithm

Final parameters: $(\mathbf{0}, \mathbf{0}, \mathbf{0}, \mathbf{0})$

43.75

40.95

1186

6

$(-0.0011,-0.0087,-0.794,-0.01)$

The stress distribution in the initial and optimized structure is presented at Figure 7(a) 


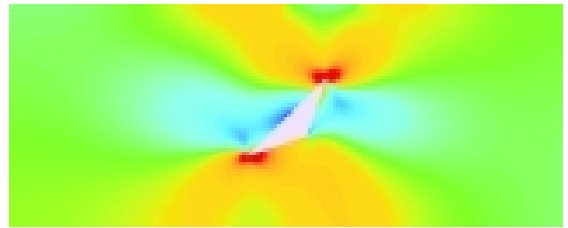

(a) before optimization

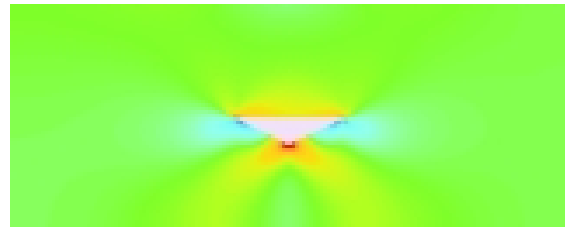

(b) after optimization $\int_{\Omega} \sigma_{e q}^{3} d \Omega$

Figure 7: Distribution of th equivalent stress (Example 4)

\subsection{Identification}

For identification of shape and location of the voids we used the functional

$$
J=\int_{\Gamma} h(\boldsymbol{u}, \hat{\boldsymbol{u}}) d \Gamma=\frac{1}{2} \int_{\Gamma_{0}}|\boldsymbol{u}-\hat{\boldsymbol{u}}|^{2} d \Gamma_{0}
$$

expressing the distance between the measured displacement field on the external boundary $\hat{\boldsymbol{u}}$ and the calculated one $\boldsymbol{u}$. The adjoint problem is loaded by the boundary traction $\boldsymbol{p}^{a}(x)=\boldsymbol{u}(x)-\hat{\boldsymbol{u}}(x)$

Example 5. The structure from Example 2 is considered again (Figure 3).

The actual void parameters are $(-1,0.5,2,-0.8)$. Identification was performed using two initial configuration.

\section{Start:}

$(\mathbf{0 , 0}, \mathbf{0 , 0})$

Initial value of functional (34):

Final value :

5.72516

$4.511 \mathrm{e}-05$

Calls of the objective function:

1186

Iterations of the main algorithm

127

Found location:

$(-1.11123,0.37997,-1.22047,-0.79172)$

For that initial guess obtained parameters constitute a local minimum of the objective function.

Table 8: Identification for starting point $(0,0,0,0)$

\begin{tabular}{||l|l|l|l||}
\hline Iteration & parameter & value of $J$ & calls of $J$ \\
\hline \hline 10 & $(-0.802,0.375,-0.881,-0.848)$ & 0.00082 & 94 \\
\hline 20 & $(-1.139,0.378,-0.859,-0.864)$ & 0.00020 & 186 \\
\hline 50 & $(-1.102,0.388,-1.135,-0.808)$ & $5.239 \mathrm{e}-05$ & 469 \\
\hline 100 & $(-1.113,0.380,-1.218,-0.792)$ & $4.515 \mathrm{e}-05$ & 928 \\
\hline \hline
\end{tabular}




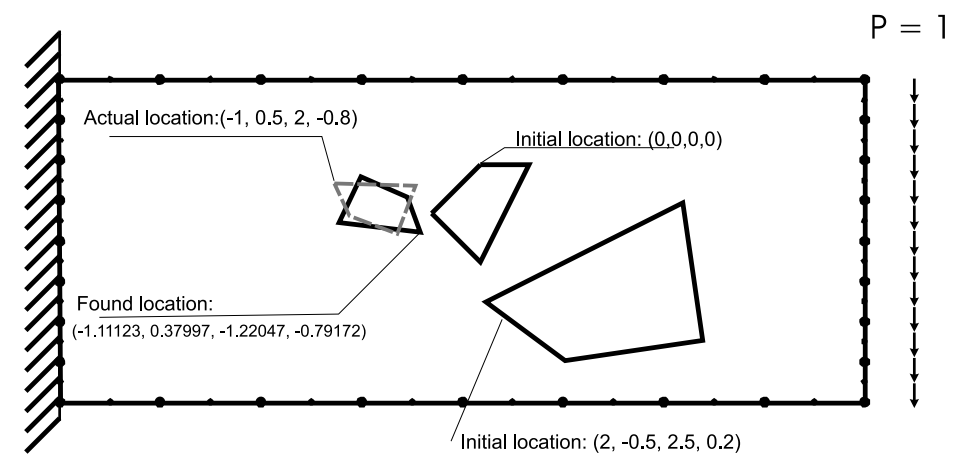

Figure 8: Identification of void (Example 5)

For another starting point we obtain the global minimum:

Start:

Initial value of functional (34) :

Final value :

Calls of the objective function:

Iterations of the main algorithm

Found location:
$(2,-0.5,2.5,0.2)$

7.02

$4.511 \mathrm{e}-17$

1902

202

$(-1.0,0.5,2,-0.8)$

Table 9: Identification for starting point $(2,-0.5,2.5,0.2)$

\begin{tabular}{||l|l|l|l||}
\hline Iteration & parameter & value of $J$ & calls of $J$ \\
\hline \hline 10 & $(-1.021,0.216,2.888,-0.793)$ & 0.00138 & 103 \\
\hline 20 & $(-1.178,0.383,2.493,-0.923)$ & 0.00048 & 199 \\
\hline 50 & $(-1.033,0.487,2.143,-0.859)$ & $1.04 \mathrm{e}-05$ & 483 \\
\hline 100 & $(-1.0006,0.5003,2.003,-0.801)$ & $4.83 \mathrm{e}-09$ & 946 \\
\hline \hline
\end{tabular}

Example 6. A stress concentrator in the form of a triangular void in the structural element (Figure 6 ) is being identified.

The actual configuration of the void is given by the vector $(0.2,-1.5,2,-0.6)$. Identification was performed starting from two initial points.

\section{Start:}

Initial value of functional (34) :

Final value :

Calls of the objective function:

Iterations of the main algorithm

Found location: $(\mathbf{0 ,}, \mathbf{0}, \mathbf{0}, \mathbf{0})$

0.0685

$1.655 \mathrm{e}-06$

597

59

(-0.067, -2.117, -1.148, -0.605) 


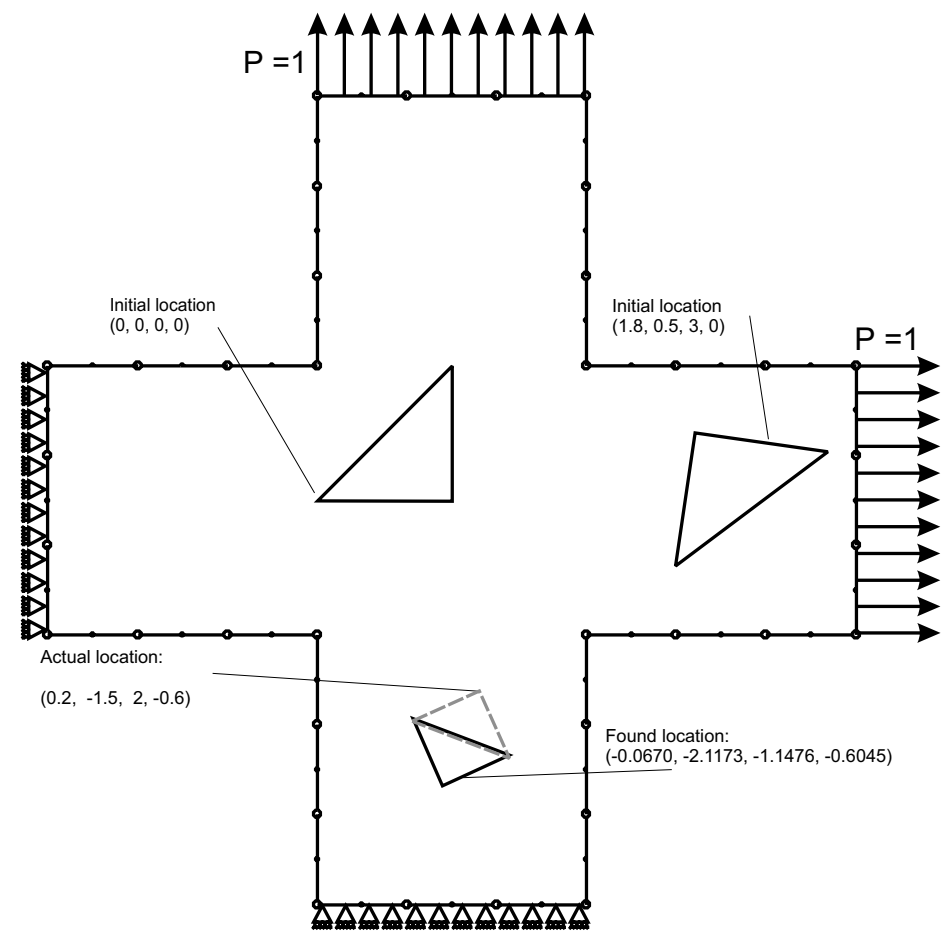

Figure 9: Structural element: identification of triangular void

Table 10: Structural element: Identification for starting point $(0,0,0,0)$

\begin{tabular}{||l|l|l|l||}
\hline Iteration & parameter & value of $J$ & calls of $J$ \\
\hline \hline 10 & $(0.017,-1.919,-1.155,-0.643)$ & 0.00033 & 119 \\
\hline 20 & $(-0.042,-2.006,-1.102,-0.591)$ & $1.35 \mathrm{e}-05$ & 221 \\
\hline 50 & $(-0.065,-2.111,-1.143,-0.604)$ & $1.70 \mathrm{e}-06$ & 518 \\
\hline \hline
\end{tabular}

The found location is at a local minimum of the functional $J$.

For another starting point we attain the global minimum:

\section{Start:}

Initial value :

Final value :

Calls of the objective function :

Iterations of the main algorithm:

Found location: $(\mathbf{1 . 8}, 0.5,3,0)$

0.04060742

$6.90973 \mathrm{e}-17$

974

95

$(0.2,-1.5,2,-0.6)$

Other attempts of identification were also performed for various starting points and for different load conditions. Many of them ended in a local minimum of the functional. 
Table 11: Structural element: Identification for starting point $(1.8,0.5,3,0)$

\begin{tabular}{||l|l|l|l||}
\hline Iteration & parameter & value of $J$ & calls of $J$ \\
\hline \hline 10 & $(0.194,-0.315,2.236,-0.345)$ & 0.00038 & 114 \\
\hline 20 & $(0.123,-0.894,2.233,-0.502)$ & 0.00011 & 221 \\
\hline 30 & $(0.192,-1.491,2.018,-0.6003)$ & $6.1 \mathrm{e}-7$ & 329 \\
\hline 50 & $(0.199,-1.496,2.001,-0.5999)$ & $1.70 \mathrm{e}-08$ & 529 \\
\hline
\end{tabular}

\section{Conclusions}

In the present work, a new method of shape sensitivity analysis for stress concentrators based on BEM is presented. This method allows for avoiding numerical difficulties arising from the presence of stress concentration in the vicinity of geometry singularities. In many numerical tests this method proved to be able to give reliable results for many types of functionals depending on mechanical quantities. These tests have shown that the accuracy of computations is much higher for functionals of the form of the boundary integral than the volume integral. For functionals expressed by the volume integral the accuracy is considerably worse, and the time of calculation is much larger. This is caused by the necessity of solving the complicated adjoint system and the fact, that calculation of the volume integral is much more time-consuming than the boundary one. However, theorems 1 and 2 allow to overcome these drawbacks for some types of functionals. It has been also shown, that the presented method of sensitivity analysis can be successfully applied in optimization and identification algorithms.

\section{References}

[1] T. Burczyński, Polch E.Z. Sensitivity analysis of crack using boundary and pathindependent integrals, proc. int. symp. on bem. In Inverse Problems in Engineering Mechanics, Proc. of the 2nd Int. Symposium on Inverse Problems. Technical University of Braunschweig, 1993.

[2] T. Burczyński, Polch E.Z. Path-independent and boundary integral approach to sensitivity analysis and identification of cracks. In Inverse Problems in Engineering Mechanics, Proc. of the 2nd Int. Symposium on Inverse Problems. ISIP'94, 1994.

[3] H.D. Bui. Dual path-independent integrals in the boundary-value problems of cracks. Engng. Fract. Mech., 6:287-296, 1974.

[4] T. Burczyński. Stochastic boundary elements to analysis, shape design sensitivity and identification problems. In Computational Stochastic Mechanics, chapter 25, pages 569-593. Cheng, A.H-D. and Yang, C.Y. (eds), 1993. 
[5] T. Burczyński. Stochastic boundary element approach to shape design sensitivity analysis. In Design Sensitivity Analysis, pages 68-92. M. Kleiber and T. Hisada, 1993.

[6] M. Habarta. Shape sensitivity analysis of structures with stress concentrators using Boundary Element Method. PhD thesis, Silesian University of Technology, Gliwice, 2000. (in Polish).

[7] C.A. Brebbia, J. Dominguez. Boundary Elements An Introductory Course. Computational Mechanics Publications, 1989.

[8] E.Z. Polch, T. Burczyński, M. Habarta,. Structural and Multidisciplinary optimization, chapter Boundary element formulation for sensitivity analysis and detection of cracks and voids., page 537-542. Pergamon, 1995.

[9] T. Burczyński, M. Habarta. Boundary anad path independent integrals in sensitivity analysis of voids. 1995.

[10] K. Dems, Z. Mróz. On a class of conservation rules associated with sensitivity analysis in linear elasticity. Int. J. Solids and Structures, 22:737-758, 1986.

[11] J.R. Rice. A path-independent integral and the approximate analysis of strain concentrations by notches and cracks. J. Appl. Mech, 35:379-386, 1968.

[12] T. Burczyński,. Path-independent integral approach to sensitivity analysis and identification problems associated with the singular and quasi-singular boundary variations. In Proc. IABEM-92 Int. Symp. on BEM. University of Colorado, 1992.

[13] G. Kokot T. Burczyński, M. Habarta,. Inverse Problems in Engineering: Theory and Practice. ASME, chapter Coupling of boundary elements and path-independent integral in generalized shape optimization and defect identification. 1998.

[14] D. Vasilopoulos. On the determination of higher order terms of singular elastic stress field near corners. Numer.Math, 53:51-95, 1988.

[15] M.L. Williams. Stress singularities resulting from various boundary conditions in angular corners of plates in extension. J.Appl.Mech., 19:526-528, 1952. 